\title{
LE LEXÈME SLOVÈNE TUDI ET SES ÉQUIVALENTS FRANÇAIS'
}

Cet article se propose d'analyser tout d'abord le lexème slovène tudi, de passer ensuite en revue les différents équivalents français de ce dernier, puis d'analyser l'équivalent le plus fréquent, à savoir l'adverbe de phrase aussi, pour enfin conclure avec une analyse contrastive quant au fonctionnement du lexème slovène et de son équivalent français aussi.

\section{LE LEXÈME TUDI}

Le lexème slovène tudi est caractérisé par deux fonctions: il assure une macrofonction au niveau du texte tout en assumant une microfonction dans la phrase où il apparaît. Il joue en effet le rôle de connecteur (avec forte connexion au contexte gauche ou forte connexion au contexte droit) tout en étant un moyen de mise en relief de l'élément auquel il se rapporte:

Tudi Peter je lačen.

(fr.: Peter aussi a faim.)

Cet énoncé renvoie inévitablement au contexte gauche et implique que »quelqu'un d'autre que Peter a faim«, tout en mettant l'accent sur l'un des éléments de la phrase, en l'occurence »Peter«.

Soulignons que la mise en relief opérée par tudi peut être simple ou renforcée (auquel cas il prend la valeur de celo - fr. même). A l'écrit, seul le contexte gauche permet de déterminer s'il s'agit d'une mise en relief simple ou renforcée, alors qu'à l'oral la prosodie peut, à elle seule, orienter l'interprétation.

Sémantiquement, il exprime de manière générale l'addition. Il peut, dans certains cas, exprimer l'identité.

Ces deux cas de valeur sémantique ont une incidence directe sur le niveau syntaxique: quand tudi exprime l'identité ou la répétition, son noyau se trouve dans le sujet (ou constitue le sujet réel de la phrase) ou dans un complément circonstanciel de lieu/temps:

Tudi Peter govori francosko.

(fr. Peter aussi parle le français.)

présupposé: quelqu'un d'autre que Peter parle le français, donc ce qui a été affirmé pour Peter l'a déjà été pour quelqu'un d'autre dans le contexte gauche.

conclusion: Peter et cette autre personne parlent la même langue.

1 Le présent article résume la thèse de 3ème cycle Skladenjsko vedenje francoskih ustreznikov slovenskega člen$k a \underline{t u d i}$, soutenue le 18 novembre 1999 à la Faculté des Lettres de Ljubljana devant le jury suivant: prof. J. Orešnik (président), prof. V. Pogačnik (directeur de thèse), prof. B. Pogorelec (membre). 
Tudi danes sem šla v kino.

(fr. Aujourd'hui aussi je suis allée au cinéma.)

présupposé possible : hier je suis allée au cinéma.

conclusion: hier et aujourd'hui j'ai fait la même chose - je suis allée au cinéma.

Par contre, quand le noyau de tudi se trouve dans les autres constituants de la phrase, il exprime l'addition:

Peter govori tudi francosko.

(fr. Peter parle aussi le français.)

présupposé: Peter parle d'autres langues que le français.

conclusion: Peter parle l'anglais, l'allemand et le français.

\section{TYPOLOGIE DES ÉQUIVALENTS FRANÇAIS}

Passons aux équivalents français du lexème slovène tudi répertoriés dans le corpus choisi, constitué à partir des exemples trouvés dans le roman slovène de Vladimir Bartol Alamut et dans la traduction française de ce dernier.

Les équivalents sont non seulement très nombreux mais aussi très variés (l'énumération est en ordre décroissant en fonction du nombre d'occurence, du plus fréquent au moins fréquent): aussi, même, également, à son tour, c'est qui/que, et, d'ailleurs, encore, donc, ne pas tarder à, surtout, quant à, voilà, y compris, de plus, jusqu'à, sur quoi, après tout, promptement, de son côté, non moins que les précédents, imiter, sans oublier, pour ne citer que, finir par, s'empresser de.

La variété des solutions proposées peut néanmoins s'expliquer en fonction des caractéristiques de tudi établies dans la première partie de cet article: chaque équivalent peut en effet être déterminé comme connecteur, comme moyen de mise en relief ou comme combinaison des deux fonctions inhérentes à tudi.

Dans le cas d'une combinaison des deux fonctions, il convient de remarquer que la valeur prise par chacune d'elle est susceptible de variation. Le lexème tudi en tant que connecteur peut présenter soit une forte connexion au contexte gauche, soit une forte connexion au contexte droit, alors qu'en tant que moyen de mise en relief il convient de distinguer entre la mise en relief simple et la mise en relief renforcée.

CONNECTEUR (à forte connexion gauche) et MISE EN RELIEF (simple) valeur sémantique: identité/répétition niveau syntaxique: noyau en fonction sujet (ou sujet réel) ou c.c. de lieu/temps

- tudi: aussi (aussi, moi/toi/lui... aussi)

"Tudi moj gospodar se je jokal, ko me je prodal," je dejala Zajnab. (p. 23)

"Mon maître aussi a pleuré en me vendant," dit Zaïnab. (p. 22)

Pomislil je na umorjenega vezirja. Spreletelo ga je, da bo moral tudi sam. umreti. (p. 529)

Il pensa à son vizir assassiné. Il sut alors qu'il devait mourir, lui aussi. (p. 524) 
En cas de négation, aussi devient non plus:

Vročina je pritisnila in voda nam bo pošla. Tudi živil ni zadosti. (p. 207)

La chaleur sévit et l'eau va manquer. Les vivres non plus ne sont pas en quantité suffisante. (p. 204)

\section{- tudi: également}

Meni se zdi njegova zamisel blazna. Toda blazen se mi je zdel tudi njegov načrt, kako se polastiti Alamuta. (p. 276)

Son idée me semble folle. Mais son plan pour s'emparer d'Alamut m'avait également paru fou. (p. 273)

- tudi: encore

$\mathrm{Da}$, to moram pripomniti: pri tem se je prav tako dvoumno in zvito hahljal kot vselej, ko je bril iz nekoga svoje norčije. Seveda sem mislil, da se tudi to pot šali in da se spodobi, če se mu smejem. (p. 156)

Le drôle de l'histoire est qu'il eut recours, en cette bizarre occurence, à ce ton ambigu et facétieux qu'il prenait d'ordinaire lorsqu'il avait quelque plaisanterie à servir: si bien que cette fois encore je crus bon de ne pas le prendre au sérieux et d'en rire. (p. 152)

Cet équivalent apparaît, dans la plupart des cas, en combinaison avec un élément anaphorique (adjectif ou pronom démonstratif)

- tudi: à mon/ton/son... tour

Abdur Ahman je najprej zapadel v sen. Džafar se je še nekaj časa upiral. Končno je tudi niega premagalo. (p. 463)

Abdur Ahman fut le premier à s'endormir. Djafar résista encore quelque temps, se retourna lourdement sur le côté, puis céda à son tour au sommeil. (p. 458)

- tudi: de mon/ton/son... côté

Sara se je izogibala in tudi Halimi je bilo nerodno, če sta se srečali. (p. 110)

Sara l'évitait et elle était, de son côté, gênée de la rencontrer. (p. 106)

\section{- tudi: non moins que les précédents}

Veliko zagonetnejši so magični načini smrti, ki jih imenujemo tudi medicinske/.../ Tudi ti nauki so navdali Ibn Tahirja $z$ velikim začudenjem. (p. 85) Restent les morts magiques, appelées aussi médicales, qui sont bien plus problématiques. /.../ Ces enseignements surprenaient Ibn Tahir non moins que les précédents. (p.82) 
CONNECTEUR (à forte connexion gauche) et MISE EN RELIEF (renforcée) valeur sémantique: identité, répétition

niveau syntaxique: noyau en fonction sujet (ou sujet réel) ou c.c. de lieu/temps

\section{- tudi: même}

Včasih je ta ali oni dai pa tudi sam Abu Ali obiskal ujetnike. (p. 393)

De temps en temps, un dey ou un autre, parfois même Abu Ali en personne, rendait visite aux prisonniers. (p. 389)

»Ni dolgo tega, ko je bil še čisto podoben mladi mačici. Pitale smo ga s kozjim mlekom in tudi zdai ga ne hranimo z mesom, da ne bi podivjal.« (p. 47)

"Il n'y a pas bien longtemps il ressemblait encore à un jeune chat; nous l'avons nourri au lait de chèvre, et même maintenant, nous nous gardons bien de lui donner le moindre morceau de viande, de peur qu'il ne devienne féroce.« (p. 45)

Le cas suivant est quelque peu particulier car la valeur sémantique de répétition est prise en charge par la sémantique du verbe, alors que la mise en relief "disparaît" dans la traduction française:

Šla je k Sulejki in jo božala po laseh. Tudi druge so prišle in jo skušale potolažiti. (p. 364)

En s'approchant de Suleikka, elle lui caressa les cheveux. Les autres l'imitèrent, s'efforçant chacune à sa façon de consoler l'infortunée. (p. 360)

En conclusion à cette partie traitant du connecteur à forte connexion au contexte gauche, les constatations suivantes s'imposent:

1. Les équivalents appartiennent aussi bien à la classe des morphèmes grammaticaux (aussi, également, même...) qu'à celle des morphèmes lexicaux (à son tour, de son côté....).

2. Parmi ces équivalents, les morphèmes grammaticaux (qui sont en fait des adverbes de phrase) participent à l'organisation et à la distribution en thème-rhème, rhématisant toujours le noyau auquel ils se rapportent, thématisant de par la même tous les autres éléments. Le thème de l'énoncé où figure tudi et le contexte gauche concerné entretiennent un lien de synonymie très fort:

"Srečen sem, da te morem sprejeti kot prvi na gradu Alumutu, « je dejal.

"Hvala, tudi mene veseli, « je odvrnil Abul Fazel. (p. 152)

»Je suis heureux d'être le premier à t'accueillir au château d'Alamut!«, dit-i1. »Merci, je m'en réjouis aussi, « répondit Abul Fazel /.../ (p. 148)

»Ali trgovec/.../, ko so me odpeljali, je glasno zajokal. Jokala sem tudi jaz.« (p.23) $»$ Le brave Ali /.../, quand on m'emmena, fondit en larmes. Je pleurais moi aussi.« (p. 22)

3. Les morphèmes lexicaux apparaissent presque toujours en combinaison avec un élément anaphorique (au sens large du terme): 
Abdur Ahman je najprej zapadel v sen. Džafar se je še nekaj časa upiral. Končno je tudi njega premagalo. (p. 463)

Abdur Ahman fut le premier à s'endormir. Djafar_résista encore quelque temps, se retourna lourdement sur le côté, puis céda à son tour au sommeil. (p. 458)

CONNECTEUR (à forte connexion au contexte droit) et MISE EN RELIEF (simple) valeur sémantique: addition

niveau syntaxique: noyau en fonction de non-sujet (ou de non-sujet réel) et non-c.c. de lieu/temps

\section{- tudi: aussi}

Imel je dve hčeri, /... /. Tudi sina je imel. (p. 22)

Ce brave homme avait deux filles /... /. Il avait aussi un fils. (p. 22)

Tudli et aussi apparaissent aussi dans les expressions ne samo... ampak/marveč tudi: non/pas seulement ... mais aussi

Stotnik Minučeher jih ni vzgajal samo v vojaških spretnosti, marveč jih je učil tudi zemliepis. (p. 121)

Le capitaine Minutchecher ne les entraînait pas seulement à la routine militaire, il leur enseignait aussi la géographie. (p. 117)

\section{- tudi: également}

" Toda vojaki so, kot si videl, izurjeni, da je veselje, in tudi z živežem in vojnimi potrebaščinami smo dobro preskrbljeni.« (p. 153)

"Mais comme tu vois, ce sont là des soldats magnifiquement entraînés, et nous sommes également bien pourvus en vivres et en matériel.« (p. 149)

- tudi: et

Učenci so drgetali od vročične napetosti. Njihov nemir se je prenesel tudi na živali, ki so pod njimi nestrpno hrzale. (p. 202)

Les jeunes gens tremblaient visiblement de tension fièvreuse, et leur angoisse se communiquait aux bêtes qui hennissaient avec impatience. (p. 199)

- tudi: de plus

Toda nihče o tem ne govori in tudi izpraševati je prepovedano. (p. 39)

/... / mais personne n'en parle, et de plus il est interdit de poser des questions sur ce sujet. (p. 38)

- tudi: sans oublier

Ibn Tahir je vzel svoje stvari s seboj. Tudi pesmi. (p. 493)

Le garçon rassembla ses affaires - sans oublier ses poèmes. (p. 488) 


\section{- tudi: y compris}

»Kar se pa mojega primera tiče, moraš upoštevati, da sem filozof in da cenim predvsem tisto, kar lahko otipam. To pa se po eni noči ne bo bog ve kaj spremenilo.« Abu Ali se je smejal. »Tudi stališče, « je rekel. (p. 362)

"Mais en ce qui concerne mon cas, tu ne dois pas oublier que je suis un philosophe et que j'apprécie avant tout ce que je peux toucher. Une seule nuit ne suffira pas à y changer grand-chose. $\mathrm{Y}$ compris le point de vue, « nota Abu Ali. (p. 358)

\section{- tudi: d'ailleurs}

Stari oče ga je skušal ukrotiti s palico in s trdim postom. Toda Hosein ni odnehal. Njemu in vsakomur se je postavil po robu, če so ga ovirali pri izživljanju njegovih strasti. Stari oče je bil tudi prvi, ki ga je Hosein smrtno zasovražil. (p. 511) Le grand-père avait essayé de dompter le petit rebelle à coups de bâton, et en le privant quasiment de nourriture. Mais le jeune enragé ne s'amendait pas et refusait de plier devant quiconque s'opposait à la satisfaction de ses caprices. L'intraitable aieul avait d'ailleurs été la première victime de la haine mortelle que le gamin vouait aux siens... (p. 506)

L'addition peut, dans certains cas, prendre des valeurs particulières:

- sur l'axe temporel (addition simple)

\section{- tudi: sur quoi}

»Pojdi zdaj, stari prijatelj,« je dejal Hasan in objel reisa okrog ramen. /.../ Pomignil je tudi Abu Aliju in oba sta ga zapustila. (p. 177)

"Va maintenant, vieil ami, « conclut Hassan en entourant de son bras les épaules du raïs, ... Sur quoi il fit signe à Abu Ali et les deux vieillards le quittèrent. (p. 173)

\section{- tudi: ne pas tarder à}

Zdaj sta prišla tudi Asad in Adi in kmalu za njima še Mohamed in Mustafa. (p. 105)

Asad et Adi ne tardèrent pas à les rejoindre, suivis de Mohammad et de Mustafa. (p. 102)

\section{- tudi: s'empresser de}

»Jaz bi hotela biti poleg tebe, « je dejala Džada. »Jaz tudi, jaz tudi.« Vse so izrazile isto željo. (p. 248)

»Je voudrais être à côté de toi, « fit Djada. »Moi aussi« fit une autre et toutes s'empressèrent d'exprimer le même désir. (p. 245) 
- tudi: promptement

Obsodili so ga tajno na smrt in obsodbo tudi izvršili. (p. 51)

Le bonhomme fut donc condamné à mort, et la sentence promptement exécutée. (p. 50)

Sur l'axe temporel, l'addition peut prendre une valeur particulière, à savoir la finalité:

\section{- tudi: finir par}

Po kakšnih desetih verzih so izčrpale svojo iznajdljivost in ostali sta samo še Fatima in Zajnab, ki sta zagrizeno vztrajali, dokler tudi njima ni odreklo. (p. 36)

Au bout d'une dizaine de vers, la plupart avait épuisé leur faculté d'invention; seules s'affrontaient encore, non sans un bel acharnement, Fatima et Zaïnab - lesquelles finirent malgré tout par crier grâce. (p. 34)

- sur l'axe argumentatif (exprimant la finalité):

- tudi: donc

Kajti one, ki so bile s Fatimo in Sulejko, so ostale v svojih paviljonih. Tudi Mirjam je spala sama. (p. 364)

Celles qui avaient suivi Fatima et Suleïka étaient restées dans leur pavillon. Myriam dormit donc seule. (p. 361)

- tudi: après tout

"Bila sem $\mathrm{v}$ haremu in tam je bilo treba neprestano glumiti in se potvarjati. /.../ Igrati v teh vrtovih hurije tudi ne bo pretežko." (p. 243)

"Moi, j'ai fréquenté les harems, et je m'entends à jouer la comédie. /.../ Je suis sûre qu'il ne doit pas être bien compliqué, après tout, de jouer aux houris dans ces jardins." (p. 240)

CONNECTEUR (à forte connexion au contexte droit) et MISE EN RELIEF (renforcée)

\section{- tudi: même}

"Na našem otoku pa imamo govedo, velblodico, štiri konje in nekaj osličev. Samo pri nas so tudi mačke in psi." (p. 103)

"Tu pourras voir encore dans l'île un troupeau de bétail, un petit chameau, quatre chevaux, et quelques ânes. Il y a même des chiens et des chats." (p. 99) 


\section{- tudi: surtout}

$\mathrm{Da}$, bila je grešnica, zavržena in pogubljena. Izgubila je tudi Mirjamino naklonjenost. (p. 109)

Oui, elle était pécheresse, réprouvée... Elle avait surtout perdu la sympathie de Myriam. (p. 105)

\section{- tudi: quant à}

Abu Ali je ponorel od ljubosumnosti. Najprej je zaklal zapeljivca, potem še nezvesto ženo. Sovražil pa je tudi Habibo, ki mu je bila izdala njeno nezvestobo. $V$ prvem srdu je naložil njo in otroka na velblode in jih odpeljal v Basro. Tam jih je prodal kot sužnje. (p. 276)

Fou de jalousie, il avait tué, dans l'ordre, le séducteur, puis l'épouse infidèle. Quant à Habiba, qui lui avait révélé son infortune, il l'expédia dans l'instant avec ses deux enfants, pour calmer sa colère, par la première caravane de Basra /.../ où il les fit vendre comme esclaves. (p. 273)

- tudi: jusqu'à

Ko je tolmacil koran, se je spuščal tudi v modroslovna razglabljanja, govoril je o drugih verstvih, prikazal učencem osnove krščanstva, židovstva in poleg raznih poganstev tudi skrivnostni indijski nauk, ki ga je bil oznanjal Budha. (p. 122) Commentant le Coran, il en approfondissait les prolongements philosophiques, mais n'hésitait pas à traiter aussi des autres religions; il exposait aux élèves les fondements du christianisme, du judaīsme, leur décrivait les différents visages du paganisme, et jusqu'aux mystères de la doctrine enseignée dans l'Inde par le Bouddha. (p. 119)

En conclusion à la connexion forte au contexte droit, les constatations suivantes s'imposent:

1. Les équivalents français peuvent être répartis en deux groupes: les morphèmes grammaticaux (aussi, également, et, de plus...) et les auxiliaires aspectuels (finir par, ne pas tarder à...).

Les morphèmes grammaticaux assurent les deux fonctions, alors que les auxiliaires aspectuels apparaissent avec un élément anaphorique pour couvrir adéquatement le lexème tudi.

2. D'un point de vue sémantique, le dénominateur commun à tous ces équivalents est l'addition, au sens large du terme. Elle peut, entre autres, avoir lieu sur l'axe temporel et l'axe argumentatif, prenant dans certains cas une valeur particulière: la finalité.

De plus, le lien entre l'énoncé où apparaît tudi et le contexte gauche est, d'un point de vue sémantique, beaucoup moins étroit que dans le cas de la forte connection à gauche (exprimant l'identité ou la répétition), car le lexème slovène, 
bien que contribuant aux principes de la cohésion, assure surtout la progression et moins le renvoi anaphorique:

"Toda vojaki so, kot si videl, izurjeni, da je veselje, in tudi z živežem in vojnimi potrebščinami smo dobro preskrblieni." (p. 153)

"Mais comme tu vois, ce sont là des soldats magnifiquement entraînés, et nous sommes également bien pourvus en vivres et en matériel." (p. 149)

3. Au niveau syntaxique (pour les équivalents faisant partie des adverbes de phrase), le noyau peut apparaître dans tous les constituants de la phrase, à l'exception du sujet et du c.c. de temps/lieu.

4. Quant à la mise en relief, elle est moins présente dans le cas des auxiliaires aspectuels que dans les autres cas de figure.

Dans certains cas, on assiste dans la traduction française à la décomposition des deux fonctions de tudi:

- tudi: c'est ... qui/que + élément anaphorique (pronom/adjectif démonstratif)

Brez besede, podoben avtomatu, jo je objel in jo prižel nase. Prav tako nezavedno in topo se je je tudi polastil. (p. 306)

Sans un mot, comme un automate, il se mit à l'embrasser. Puis il l'attira violemment à lui. Les caresses qu'ils échangèrent ensuite ne parurent pa dissiper entièrement l'hébétude où il restait plongé. C'est dans cet état de semi-conscience qu'il la posséda. (p. 303)

\section{- tudi: encore + élément anaphorique (pronom/adjectif démonstratif)}

Deklice so prestrašene poskakale na noge... Kmalu so bile tudi deklice v tem paviljonu na nogah. Apama je odšla s skopljenci, da bi pripeljali tudi dekleta s tretjega vrta. (p. 381)

Les jeunes filles, terrorisées, furent debout dans l'instant... La vieille femme accompagna les eunuques dans leur visite aux pavillons voisins, où les dormeuses, là encore, furent promptement tirées du lit. (p. 376)

Dans l'exemple suivant, seule une des deux fonctions de tudi est présente dans la traduction française, à savoir la mise en relief:

Jusuf je ves prestrašen odprl oči. /.../ Glej, tudi Sulejman se je prebudil. (p. 368)

Yusuf ouvrit des yeux effrayés. /.../ Tiens, voilà notre Suleïman réveillé. (p. 364)

La connexion au contexte gauche est beaucoup moins forte dans la traduction française que dans le texte slovène.

\section{L'ADVERBE DE PHRASE $A U S S I$}

L'équivalent français le plus fréquent du lexème slovène tudi est l'adverbe de phrase aussi (qui apparaît dans plus d'un tiers du corpus). L'analyse de aussi (le cor- 
pus ayant été constitué à partir d'exemples fournis par la base de données du TLF) a permis la conclusion suivante: aussi renferme deux fonctions - celle de connecteur et celle de moyen de mise en relief - , c'est-à dire les mêmes que celles caractérisant le lexème slovène tudi: .

Pierre aussi a un frère.

Présupposé (renvoi au contexte gauche): quelqu'un d'autre que Pierre a un frère. Mise en relief de l'élément "Pierre"

Sémantiquement il exprime de manière générale l'addition, tout en pouvant, dans certains cas, traduire la répétition ou l'identité. Cette valeur sémantique particulière est observée dans certains cas de figure syntaxiques: quand le noyau de aussi occupe la fonction de sujet (ou de sujet réel) ou de c.c. de lieu/temps:

Besson ouvrit le cahier et commença à lire; ce n'était pas facile, parce que les mots n'avaient pas été écrits au crayon et s'étaient effacés depuis une vingtaine d'années. L'orthographe aussi laissait à désirer, et il fallait s'y prendre à deux ou trois reprises pour comprendre le sens des phrases. (TLF, 544)

Et pourtant Tavernier, cet infatigable explorateur de l'orient, affirme que le faste du roi soleil pâlit auprès de celui du grand mongol qu'un autre souverain, Auguste de Saxe, essaiera de copier en ruinant ses sujets. En France aussi la misère populaire s'accroît, tandis que l'insouciant Philippe d'Orléans achète le régent que Louis XVI portera à l'ouverture des Etats-généraux. (TLF, 63)

Dans les autre positions syntaxiques, on est en présence de l'addition, au sens large du terme:

Dans le domaine de la biologie animale, le XIXème siècle a été la grande époque de l'anatomie comparée. Il a aussi fondé l'anatomie générale, édifiée sur la notion de tissus. (TLF, 244)

L'adverbe de phrase aussi est par ailleurs très mobile à l'intérieur de la phrase. Il peut occuper différentes positions, à l'exception de la position initiale en tête de phrase (cette position est "réservée" à l'expression de la conséquence et demande l'inversion du sujet):

(1) Paul aussi a mangé des gâteaux dans le salon.

(2) Paul a aussi mangé des gâteaux dans le salon.

(3) Paul a mangé aussi des gâteaux dans le salon.

(4) Paul a mangé des gâteaux aussi dans le salon.

(5) Paul a mangé des gâteaux dans le salon aussi.

Les phrases (2) et (3) sont ambiguës: sans contexte, il est difficile de déterminer avec certitude le noyau. Cela pourrait aussi bien être Paul que des gâteaux. Il est vrai que normalement aussi suit l'élément auquel il se rapporte, mais cette règle est très peu respectée dans la pratique (dans seulement un dizième des cas). Les exemples du corpus ont montré que la position privilégiée de aussi est après le verbe ou entre 
l'auxiliaire et le participe passé (pour les temps composés), ce qui peut poser certaines difficultés d'interprétation en cas d'absence du contexte gauche, surtout si le noyau pourrait aussi bien être le sujet que le complément d'objet direct.

Il est vrai que le français dispose d'un moyen efficace en cas d'équivoque: il peut en effet recourir à moi/toi/lui ... aussi, qui a pour ainsi dire une fonction "désambiguïsante", car renvoyant toujours au sujet, quelle que soit d'ailleurs sa position dans la phrase, évitant ainsi toute interprétation erronée:

(2) Paul a lui aussi, mangé des gâteaux dans le salon.

(3) Paul a mangé, lui aussi, des gâteaux dans le salon.

\section{CONVERGENGES ET DIVERGENCES ENTRE TUDI ET AUSSI}

Tous deux assurent une macrofonction (celle de connecteur) et une microfonction (comme moyen de mise en relief).

De par leur macrofonction, ils contribuent doublement à la cohésion du texte: ils sont anaphoriques tout en participant à la progression. Leur position sur l'axe anaphore-progression n'est pas fixe. Ils sont parfois plus fortement liés au contexte gauche, parfois plus au contexte droit.

En cas de forte connexion au contexte gauche, ils prennent tous deux une valeur sémantique particulière, celle d'identité ou de répétition (forme particulière de l'addition), avec répercussion au niveau syntaxique: l'élément auquel ils se rapportent (le noyau) a la fonction de sujet (ou de sujet réel) ou de complément circonstanciel de temps/de lieu:

Tudi ona dobro pozna Seiduna. (p. 31)

Elle aussi connaît bien Seiduna. (p. 30)

En cas de forte connexion à droite, leur valeur sémantique est celle de l'addition. Sur le plan syntaxique, leur noyau se trouve en fonction de non-sujet ou de non-c.c. de temps/lieu.

Tudi medveda smo imeli. (p. 103)

Nous avions aussi un ours. (p. 99)

Leur microfonction au sein de la phrase est la mise en relief de l'élément auquel ils se rapportent respectivement et avec lequel ils forment un tout:

Ali se ga tudi žene boje? (p. 104)

Est-ce que les femmes aussi on peur de lui? (p. 101)

Malgré un très grand degré de recoupement entre tudi et aussi (ce qui explique, entre autres, que aussi soit l'équivalent français le plus fréquent dans le corpus étudié), les deux présentent néanmoins certaines divergences.

La première différence relève de la position qu'ils occupent par rapport à leur noyau. Le lexème slovène tudi précède le noyau auquel il se rapporte, par contre l'adverbe de phrase aussi, qui normalement devrait suivre le noyau, privilégie une position dans la phrase (après le verbe en cas de temps simple, entre l'auxiliaire et le participe passé en cas de temps composé), ce qui peut engendrer certaines ambiguïtés 
quant à l'interprétation. Sur 200 exemples seule une vingtaine respecte cette position (immédiatement après le noyau). La présence du contexte gauche est alors indispensable pour éviter les erreurs d'interprétation:

Izkazujem ti tudi svoje popolno zaupanje. (p. 200)

Je te témoigne aussi mon entière confiance. (p. 196)

L'exemple slovène ne présente aucune ambiguité, alors que la formulation française peut être comprise de deux manières différentes:

(1) Je te témoigne, moi aussi, mon entière confiance.

(2) Je te témoigne, en plus de mon admiration, mon entière confiance.

En fait, le contexte gauche montre qu'il s'agit de l'interprétation (2).

Il convient également de constater qu'au niveau de la microfonction, tudi peut aussi bien traduire une mise en relief simple qu'une mise en relief renforcée, alors que aussi est moins flexible, prenant en général la valeur de mise en relief simple:

Toda če ga boš kdaj videla, boš imela občutek, da pozna vse tvoje misli, tudi tiste, ki jih pred vsem svetom skrivaš. (p. 104)

Mais, si tu le vois un jour, tu auras l'impression qu'il connaît toutes tes pensées, même celles que tu crois cacher le mieux. (p. 101)

La traduction de tudi par aussi serait en effet beaucoup moins satisfaisante:

? Mais, si tu le vois un jour, tu auras l'impression qu'il connaît toutes tes pensées, celles aussi que tu crois cacher le mieux. (p. 101)

Terminons par la différence fondamentale entre tudi et aussi: bien que possédant tous deux les deux mêmes fonctions (une macrofonction - celle de connecteur - et une microfonction - celle de moyen de mise en relief), ils se différencient quant à la combinatoire de ces deux fonctions. Le lexème tudi est connecteur ET/OU moyen de mise en relief, alors que l'adverbe de phrase aussi ne peut être que connecteur ET moyen de mise en relief. Le lexème slovène peut, sans grande difficulté, se décharger de l'une de ses deux fonctions, si celle-ci est prise en charge par un autre élément de la phrase. Il arrive même que tudi se vide de ses deux fonctions pour devenir simplement explétif. L'adverbe de phrase français ne peut être, lui, choisi que s'il assume les deux fonctions qui lui sont intrinsèques, sinon il devient redondant, voire incorrect. Cette remarque concernant aussi a toute sa pertinence dans l'explication d'un grand nombre d'exemples figurant dans le corpus où il y a tout simplement effacement de tudi dans la traduction française. 
Le lexème slovène tudi et son équivalent français le plus fréquent dans le corpus choisi (le roman Alamut de V. Bartol et sa traduction française), l'adverbe de phrase aussi, sont caractérisés par les mêmes fonctions: une macrofonction (au niveau du texte), celle de connecteur, et une microfonction (au niveau de la phrase), celle de moyen de mise en relief. Tous deux participent à l'organisation thème-rhème (fonction rhématisatrice) et présentent la même valeur sémantique, l'addition, pouvant néanmoins, dans certaines positions syntaxiques, prendre une valeur particulière de l'addition, celle d'identité ou de répétition. Bien que les fonctions intrinsèques de tudi et aussi se recoupent, certaines divergences ont néanmoins été établies quant à leur emploi. Ils n'occupent pas le même emplacement par rapport à leur noyau (tudi avant le noyau, aussi après le noyau), le français dérobant souvent à cette règle en privilégiant la position après le verbe (quel que soit l'emplacement du noyau), pouvant engendrer de par la même des ambiguittés au niveau de l'interprétation.

La principale divergence réside dans la combinatoire des deux fonctions (macroet microfonction): le lexème tudi peut être en effet un moyen de connexion et/ou un moyen de mise en relief, alors que l'adverbe aussi ne peut être employé que s'il est moyen de connexion et moyen de mise en relief, ne pouvant donc pas se décharger de l'une de ces fonctions.

Cette constatation a toute son importance car elle permet d'expliquer le grand nombre et la variété des équivalents français répertoriés dans le corpus: les différentes solutions peuvent en effet être déterminées à partir des différentes combinaisons possibles des fonctions inhérentes au lexème slovène tudi.

Il convient toutefois de souligner que, dans un très grand nombre de cas (dans environ un quart du corpus), il y a tout simplement effacement du lexème tudi dans la traduction française. Ces cas de figure n'ont pas été abordés dans la présente contribution et pourront faire l'objet d'un prochain article.

\section{CORPUS}

BARTOL, V. (1988): Alamut. Ljubljana: Mladinska knjiga.

BARTOL, V. (1988): Alamut. Paris: Phébus. /traduit par.: Claude Vincenot, adapté par: J.-P. Sicre/.

TRÉSOR DE LA LANGUE FRANÇAISE

\section{BIBLIOGRAPHIE}

BEAUGRANDE, R. A., DRESSLER, W. U. (1992): Uvod v besediloslovje. Ljubljana: Park.

BRAUSSE, U. (1994): Lexikalische Funktionen der Synsemantika. Tübingen: Narr.

COMBETTES, B. (1983): Pour une grammaire textuelle: la progression thématique. Paris-Gembloux: Duculot.

ČERNELIC̆, I. (1991): Členek kot besedna vrsta v slovenskem knjižnem jeziku, v: Jezikoslovni zapiski 1991. Ljubljana: ZRC SAZU, str. 73-85.

ČERNELIČ-KOZLEVČAR, I. (1993): O delitvi členkov, v: Zbornik Slavističnega društva Slovenije 3. Ljubljana: Zavod RS za šolstvo in šport, str. 213-227. 
DUCROT, O. (1980): Les échelles argumentatives. Paris: Editions de Minuit.

DUCROT, O. (1980): Les mots du discours. Paris: Editions de Minuit.

DUCROT, O. (1984): Le dire et le dit. Paris: Editions de Minuit.

HELBIG, G. (1988): Lexikon deutscher Partikeln. Leipzig: Verlag Enzyklopädie.

LANGUE FRANÇAISE (1988), no. 77, Syntaxe des connecteurs. Paris: Larousse.

LANGUE FRANÇAISE (1989), no. 81, Structurations de textes: connecteurs et démarcations graphiques. Paris: Larousse.

LANGUE FRANÇAISE (1990), no. 88, Classification des adverbes. Paris: Larousse.

LOKAR, J. (1957/58): Vloga in mesto clenka tudi, v: JiS 1957/58. Ljubljana, str.158-161.

NØLKE, H. (1983): Problems in the semantic/pragmatic description of French adverbials like: même, aussi, surtout and seulement, v: Acta Linguistica Hafniensia, volume17, no 2. Copenhagen: The Linguistic Circle of Copenhagen, str. 157-168.

NøLKE, H. (1990): Classification des adverbes, v: Langue française, no 88. Paris: Larousse, str 3-4, 12-27.

NØLKE, H. (1993): Le regard du locuteur. Paris: Kimé.

OVEN, J. (1999): Skladenjsko vedenje francoskih ustreznikov slovenskega clenka tudi. Magistrsko delo (thèse de 3ème cycle), Faculté des lettres, Université de Ljubljana.

PERRIN-NAFFAKH, A.-M. (1996): Aussi adjonctif: de la syntaxe à la sémantique, v: Le Français moderne, LXIV, no 2, Paris: Editions CILF, str. 136-155.

RIEGEL, M. et al. (1994): Grammaire méthodique du français. Paris: PUF.

ROSSARI, C. (1994): Les opérations de reformulation. Berne: Peter Lang.

TOPORIŠIČ, J. (1974): Kratko oblikoslovje slovenskega jezika, v: SJLK, informativni zbornik. Ljubljana, str. $29-50$.

TOPORIŠIČ, J. (1976): Slovenska slovnica. Marib.or: Založba Obzorja Maribor.

TOPORIŠIČ, J. (1982): Nova slovenska skladnja. Ljubljana: Državna založba Slovenije.

TOPORIS̆IC̆, J. (1991): C̆lenki in njihovi stavčni ustrezniki, v: Seminar slovenskega jezika, literature in kulture. Ljubljana, str. 3-16.

WEINRICH, H. (1989): Grammaire textuelle du français. Paris: Didier-Hatier.

\section{Povzetek}

\section{SLOVENSKI ČLENEK TUDI IN NJEGOVI USTREZNIKI V FRANCOŠC̆INI}

Avtorica v članku obravnava slovenski členek tudi in njegove francoske ustreznike $\mathrm{v}$ izbranem korpusu (Bartolov roman Alamut v slovenskem izvirniku in francoski verziji). Členek tudi ima makrofunkcijo ( $v$ okviru besedila) in mikrofunkcijo (v okviru stavka). Njegova besediloslovna vloga namreč dvojno prispeva $\mathrm{k}$ načelu kohezivnosti znotraj besedila: poleg anaforičnosti skrbi tudi za progresijo v besedilu. Poleg besediloslovne ima členek tudi pomembno vlogo v okviru stavka oziroma povedi, to je poudarjalnost. Slovenski členek neposredno vpliva tudi na členitev po aktualnosti (rematska vloga). Glavna pomenska prvina tega členka v slovenščini je dodajalnost. Seznam ustreznikov $v$ francoščini je presenetljivo dolg in pomensko raznolik, vendar je možno vse ustreznike opredeliti kot prepletanje navezovalnosti ( $z$ različnim deležem navezovalnosti $v$ levo ali $v$ desno) in poudarjalnosti (navadne ali okrepljene). Najbolj pogosta prevodna rešitev (približno ena tretjina vseh primerov) je francoski sobesedilni prislov aussi, ki obenem najbolj pokriva tudi, saj ima isti dve temeljni funkciji kot slovenski členek (navezovalnost in poudarjalnost). Prva večja razlika med slovenskim členkom in francoskim prislovom je v njunem mestu glede na besedo, na katero se nanašata, to je glede na njuno jedro. Glavno razhajanje pa je naslednje: medtem ko tudi lahko povezuje navezovalnost in poudarjalnost ali pa izbira med njima, aussi druge t.j. alternativne možnosti ne pozna. 